\title{
La spectrométrie à écho de spins de neutrons
}

\author{
S. Pouget
}

Institut Laue-Langevin, 6 rue Jules Horowitz, BP. 156, 38042 Grenoble cedex 9, France

\begin{abstract}
Résumé. Ce cours est consacré à la technique de spectrométrie par échos de spins de neutrons (SES). Dans une première partie nous en présentons les domaines d'application. Puis, après avoir décrit le principe de la technique, nous détaillons les différentes étapes d'une expérience de SES: la préparation, les différents types de mesure possibles ainsi que le traitement des résultats. Enfin, quelques exemples de résultats obtenus par SES sont discutés.
\end{abstract}

\section{POURQUOI UTILISER LE SPECTROMETRE A ECHOS DE SPINS DE NEUTRONS (SES)?}

\subsection{Les caractéristiques}

Le spectromètre à échos de spins est, au même titre que le spectromètre à temps de vol ou le spectromètre à rétrodiffusion, un outil dédié à l'étude de la diffusion quasiélastique des neutrons. Il permet de mesurer de faibles transferts d'énergie associés aux processus de diffusion des neutrons par un échantillon.

Dans les mesures par temps de vol ou rétrodiffusion, une haute résolution en énergie est le résultat d'une détermination précise des énergies incidente et finale des neutrons. Cette monochromatisation se fait, naturellement, aux dépends du flux. Une des originalités de la technique d'échos de spins est de pouvoir combiner "mauvaise" monochromatisation (15\%) et très haute résolution en énergie (neV). Mais l'envers de la médaille est alors une mauvaise résolution en moment transféré.

La seconde originalité du SES est de mesurer directement la fonction intermédiaire de diffusion $S(Q, t)$ et non pas la fonction de diffusion en énergie $S(Q, \omega)$.

\subsection{Que peut-on donc mesurer sur un SES ?}

Dans sa configuration la plus couramment utilisée, le SES permet d'étudier la dépendance en moment transféré et en temps de la fonction intermédiaire de diffusion associée à des processus quasiélastiques. Les temps de Fourier accessibles vont de la picoseconde à la centaine de ns. Comme on peut le voir sur la figure 1, le SES permet de gagner deux ordres de grandeur de résolution en énergie $(\sim$ neV) par rapport à un spectromètre à rétrodiffusion $(\sim 0.1 \mu \mathrm{eV})$, qui lui-même donne accès à des largeurs de raies dix fois inférieures à celles observables avec un spectromètre à temps de vol $(\sim \mu \mathrm{eV})$. On peut, entre autres sujets étudiés sur les SES, citer la dynamique des polymères [1], la transition vitreuse [2], la dynamique des composés magnétiques désordonnés [3] ou encore la physico-chimie (colloïdes, microémulsions...) [4].

Mais le SES peut également être utilisé pour mesurer des largeurs de raies associées à des processus de diffusion inélastique. Un exemple en est l'étude du roton dans l'hélium 4 superfluide[5]. 


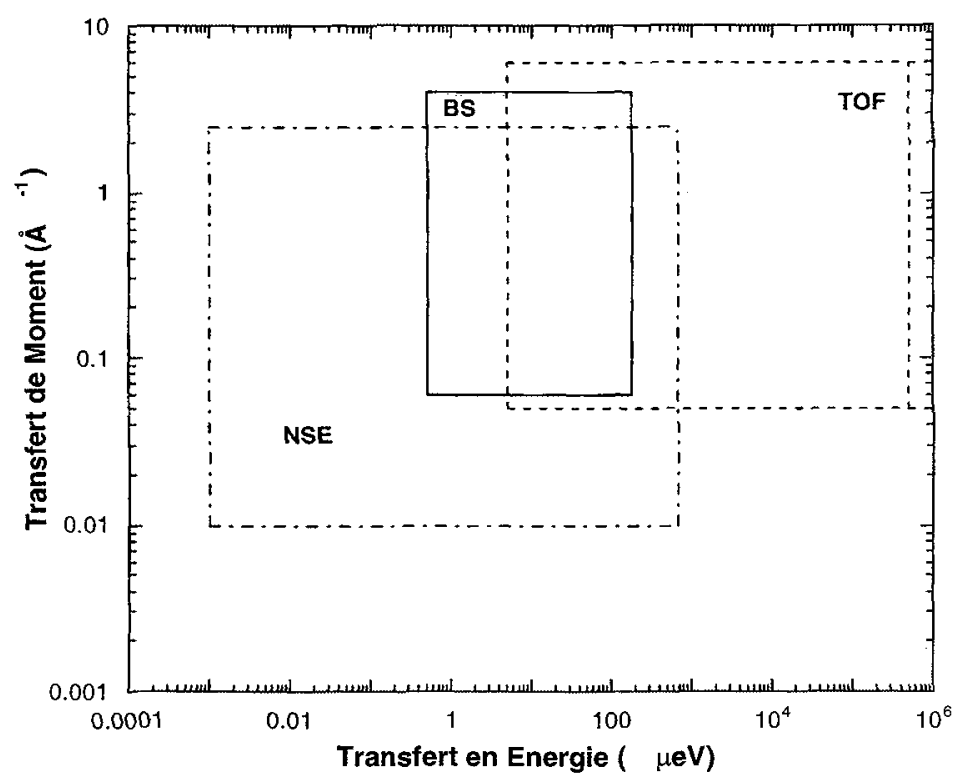

Figure 1: Domaines accessibles, en transferts d'énergie et de moment, par les trois techniques de diffusion quasiélastiques des neutrons: la spectrométrie à temps de vol (TOF), la spectrométrie en rétrodiffusion (BS) et la spectrométrie à échos de spins (NSE).

\section{PRINCIPE DU SPECTROMETRE A ECHOS DE SPINS}

\subsection{Le principe d'un spectromètre idéal}

La référence [6] présente dans le détail la théorie qui sous-tend le principe de fonctionnement d'un spectromètre à échos de spins. Nous n'en rappellerons ici que les grandes lignes.

Le point de départ est un faisceau de neutrons grossièrement monochromatisé $(\Delta \lambda / \lambda \sim 15 \%)$, qui passe à travers un polariseur.

L'état de spin $|\Psi\rangle$ d'un neutron peut se développer sur une base à deux dimensions, $\{|+\rangle,|-\rangle\}, \operatorname{Par}$ rapport à une direction donnée, par exemple l'axe $z$ du faisceau, $|+\rangle$ et $\mid->$ peuvent être vus comme les états d'un spin pointant vers $+z$ ou $-z$. Quel que soit $|\Psi\rangle$, il peut être exprimé comme une combinaison linéaire des états $\mid+>$ et $\mid->$ de la forme $\left|\Psi>=\cos \frac{\theta}{2} \exp \left(-i \frac{\varphi}{2}\right)\right|+>+\sin \frac{\theta}{2} \exp \left(i \frac{\varphi}{2}\right) \mid->$, où les angles polaires $\theta$ et $\varphi$ caractérisent la direction associée à $\mid \Psi>$ [7]. En sortie du polariseur, tous les spins sont dans le même état, par exemple $\mid+>$ : le faisceau de neutrons est polarisé. Puis les spins sont basculés dans une direction perpendiculaire à leur direction de polarisation (flipper $\pi / 2$ ) de manière à initier la précession de Larmor autour d'un champ magnétique $H$ parallèle à l'axe $z$. Soit $\left|\Psi>^{0}=\cos \frac{\theta^{0}}{2} \exp \left(-i \frac{\varphi^{0}}{2}\right)\right|+>+\sin \frac{\theta^{0}}{2} \exp \left(i \frac{\varphi^{0}}{2}\right) \mid->$ l'état d'un spin après traversée du flipper $\pi / 2$. La 
précession de Larmor a pour effet de transformer l'angle $\varphi^{0}$ en $\varphi^{0}+\varphi$, l'angle de précession $\varphi$ étant donné par

$$
\varphi=2 \pi \gamma_{L} \frac{\int H d l}{V}
$$

où $v$ est la vitesse du neutron et $\gamma_{L}$ le coefficient de Larmor.

Un spectromètre à échos de spins est caractérisé par deux aires de précession, auxquelles sont associées respectivement les intégrales de champ $\int H_{0} d l_{0}$ et $\int H_{1} d l_{1}$. Le passage d'une aire de précession à l'autre s'effectue à travers une bobine appelée flipper $\pi$, dont l'axe est perpendiculaire à la direction $z$. Elle transforme l'angle de précession $\varphi$ de chaque spin en son opposé $-\varphi$ (le spin effectue une rotation d'un angle $\pi$ autour de l'axe du flipper); l'échantillon est généralement placé près de ce flipper $\pi$. En fin de la seconde aire de précession, les spins sont ramenés parallèlement au champ magnétique (second flipper $\pi / 2$ ), ce qui stoppe la précession de Larmor. L'angle total de précession est donc donné par

$$
\varphi=2 \pi \gamma_{L}\left(\frac{\int H_{0} d l_{0}}{v_{0}}-\frac{\int H_{1} d l_{1}}{v_{1}}\right),
$$

où $v_{0}$ et $v_{1}$ sont les vitesses respectives du neutron avant et après interaction avec l'échantillon. Dans la "configuration quasiélastique", les deux intégrales de champ sont égales; aussi, si l'interaction avec l'échantillon est élastique, quelie que soit la vitesse du neutron son angle $\varphi$ associé sera nul. Les flippers $\pi / 2$ sont réglés de manière à ce qu'un spin dans l'état $|+\rangle$ se retrouve parallèle à l'un des deux vecteurs unitaires de la direction $x$, par exemple $u_{x}^{+}$, et réciproquement . Aussi, après passage à travers le second flipper $\pi / 2$, un spin auquel est associé un angle $\varphi$ nul et donc qui est parallèle à $u_{x}^{+}$, se retrouve dans l'état $1+>$. Par contre, un spin auquel est associé un angle $\varphi$ non nul se retrouve, par effet du flipper $\pi / 2$, dans un état $(\cos \varphi|+>+\sin \varphi|->)$. Un analyseur, placé devant le détecteur, ne transmet qu'un seul des deux états propres $\mid+>$ et $\mid->$. Pour simplifier nous supposerons qu'il s'agit, comme pour le polariseur, de l'état $\mid+>$. Considérons à présent le cas d'une interaction inélastique des neutrons avec un échantillon placé au voisinage du flipper $\pi$. La vitesse du neutron étant différente dans les deux aires de précession, l'angle $\varphi$ est non nul. Dans ce cas, pour une valeur donnée du moment transféré et des intégrales de champ $l_{0} H_{0}=l_{1} H_{1}$, l'intensité $I_{e c}$ mesurée par un SES idéal est la moyenne de $\cos \varphi$, pondérée par la fonction de diffusion de l'échantillon $S(Q, \omega)$. L'angle $\varphi$ est une fonction du transfert en énergie $\omega$; il peut être exprimé sous la forme $\varphi=t \omega$, où $t=\frac{\hbar \gamma_{L} l H}{m V^{3}}$. Il vient alors

$$
I_{e c}=\int \cos [\varphi(\omega)] S(Q, \omega) d \omega=\int \cos (\omega t) S(Q, \omega) d \omega
$$

Ceci n'est autre que la fonction intermédiaire de diffusion de l'échantillon $S(Q, t)$, la valeur du temps $t$ étant fixée par celle des intégrales de champ $l_{0} H_{0}=l_{1} H_{1}$.

Dans le cas d'une interaction purement élastique, l'intensité est idéalement constante quel que soit $t$, et égale à $S(Q, t=0)$.

En fait, pour un SES réel, l'intensité décroît aux temps longs c'est à dire pour des valeurs élevées des intégrales de champ, du fait de la présence inévitable d'inhomogénéités de champ le long du trajet des neutrons. Ainsi, le résultat obtenu en présence d'un diffuseur élastique est la fonction de résolution du spectromètre $R(Q, t)$. Plus généralement, quel que soit lé diffuseur, la grandeur réellement mesurée par un SES est le produit de la fonction intermédiaire de diffusion $S(Q, t)$ par $R(Q, t)$ (transformée de Fourier en énergie de la convolution de $S(Q, \omega)$ par $R(Q, \omega))$.

Pour un SES réel, les inhomogénéités de champ, les imperfections des flippers ainsi que celles du système polariseur/analyseur ont pour effet de réduire la polarisation du faisceau de neutrons. Or 
l'information relative à $S(Q, t)$ n'est portée que par la seule composante polarisée du faisceau; on ne peut donc se satisfaire d'une seule mesure.

\section{Mesure de $S(Q, t)$}

La figure 2 montre l'évolution du comptage dans le détecteur lorsque l'on varie l'intégrale de champ associée à l'une des deux aires de précession, par exemple $l_{0} H_{0}$, autour de la valeur d'équilibre $l_{0} H_{0}=l_{1} H_{1}$ (ceci est réalisé en variant le courant dans une bobine dite de phase). Les oscillations, appelées échos, sont dues à la seule partie polarisée du faisceau. Elles correspondent à la variation de l'angle de précession $\varphi$ en fonction de la différence d'intégrale de champ entre les deux aires de précession. Deux maxima successifs sont associés à des valeurs de $\varphi$ qui diffèrent de $2 \pi$. Le maximum de l'enveloppe de l'écho correspond à la valeur d'équilibre des deux intégrales de champ. La forme de cette enveloppe dépend de la monochromaticité du faisceau: elle est très étendue pour un faisceau quasimonochromatique; au contraire, si les neutrons ont des vitesses très différentes, un petit déséquilibre entre les deux aires de précession conduit à une distribution large en angles de précession $\varphi$ et donc à une diminution importante de la polarisation. Une mesure de SES consiste à mesurer quatre points déphasés de $\pi / 2$ autour du maximum de l'enveloppe d'écho, de manière à calculer l'amplitude maximale de l'écho. Cette grandeur est égale au produit $S(Q, t) * R(Q, t)$.

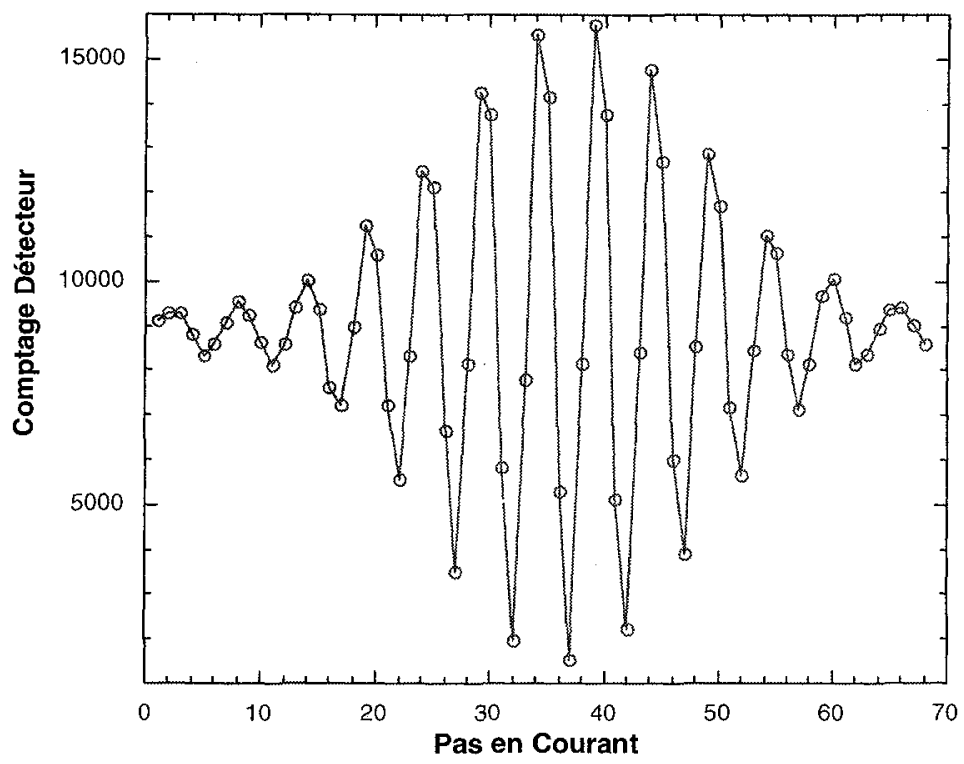

Figure 2: Evolution du comptage dans le détecteur, lorsqu'on varie le courant dans une bobine de précession. Deux maxima successifs correspondent à deux valeurs de l'angle de précession $\varphi$ qui diffèrent de $2 \pi$. Le pas en courant de précession associé est très faible (de l'ordre de $0,045 \mathrm{~A}$ à $6 \AA ̊$ ). Aussi la mesure de l'amplitude de l'écho est réalisée en variant le courant dans une petite bobine, dite bobine de phase, enroulée sur la bobine de précession principale, et pour laquelle le pas en courant est plus important (de l'ordre de $0,6 \mathrm{~A}$ à $6 \AA$ A). Pour chaque temps $t$ sondé différent, la valeur correspondante en courant de précession est fixée dans une aire et le courant est ajusté dans la seconde en réalisant un balayage en courant de la bobine de phase. 


\subsection{Un exemple de Spectromètre à Echos de Spins: IN11 à l'ILL}

La figure 3 présente les différents éléments constitutifs du SES IN11.

- Le premier rencontré par les neutrons est le sélecteur qui monochromatise le faisceau incident en ne laissant passer que les neutrons ayant la vitesse $\mathrm{V}_{0}$ désirée, avec une tolérance voisine de $15 \%$ $(\Delta v / v=15 \%$ où $\Delta v$ est la demi-largeur à mi-hauteur de la distribution des vitesses en sortie du sélecteur).

- Vient ensuite le polariseur constitué de supermiroirs de Fe-Co déposés sur un substrat de silicium pur. Un champ magnétique $H_{P}$ parallèle au faisceau incident définit la direction d'aimantation. Le polariseur transmet un seul état de spin, par exemple $1+>$, donnant ainsi un faisceau polarisé.

- Un champ magnétique parallèle à $H_{P}$, appelé champ guide, permet de conserver la polarisation entre la sortie du polariseur et l'entrée dans la première aire de précession. Celle-ci est déterminée par la traversée du flipper de Mezei $\pi / 2$, qui modifie la polarisation du faisceau de manière à la rendre perpendiculaire à $H_{P}$. Débute alors la précession des spins autour du champ magnétique de précession $H_{0}$ parallèle à $H_{P}$.

- Un second flipper, le flipper $\pi$, délimite la fin de la première aire de précession et l'entrée dans la seconde.

- La fin de la seconde aire de précession est déterminée par le passage à travers un second flipper $\pi / 2$, qui ramène les spins dans leur direction de polarisation.

- Vient enfin l'analyseur. Il est constitué de supermiroirs de CoTi déposé sur du Silicium. A la différence du polariseur, les "bons neutrons" (ici les neutrons dont le spin est dans l'état $\mid->$ ) sont réfléchis par les supermiroirs et parviennent dans le détecteur.
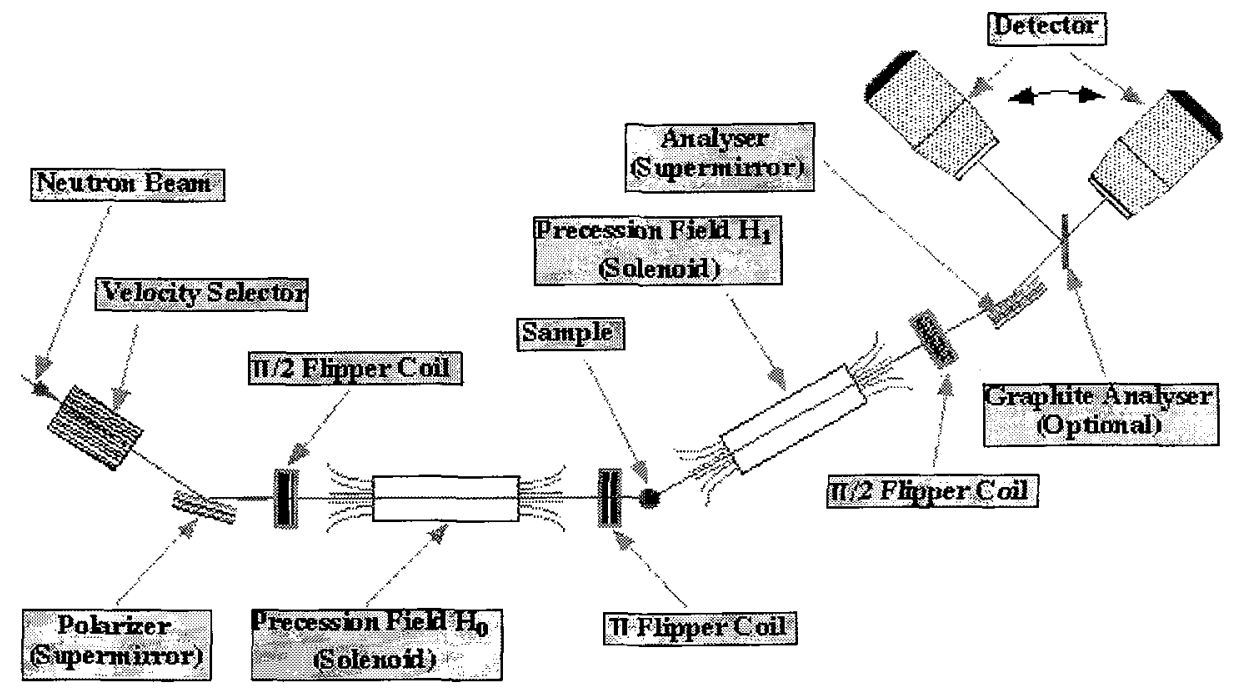

Figure 3: $\quad$ Vue schématique du Spectromètre à Echos de Spins IN11.

\section{UNE EXPERIENCE DE SES: PREPARATION ET RESULTATS}

\subsection{Détermination de $S(Q, t=0)$}

Nous avons vu qu'une mesure de SES donne accès à la grandeur $S(Q, t) * R(Q, t)$, où le temps $t$ sondé est proportionnel à l'intégrale de champ $\left(l_{i} H_{i}\right)$ d'une aire de précession, et donc non nul. Or il est intéressant 
de pouvoir comparer les valeurs mesurées de $S(Q, t)$ à la valeur $S(Q, t=0)$, intégrale sur $\omega$ de la fonction de diffusion $S(Q, \omega)$. Cette mesure est réalisée dans une configuration dite up/down, dans laquelle les spins ne précessent pas. Elle fait l'objet de ce premier paragraphe.

Considérons ici une configuration dans laquelle le faisceau de neutrons est polarisé, passe à travers deux flippers $\pi$, dont le rôle est de faire basculer les spins de l'état $|+\rangle$ vers l'état $|-\rangle$ où réciproquement, puis enfin à travers l'analyseur. Quatre mesures peuvent être réalisées dans le faisceau direct, sans échantillon: soit $I_{d d}, I_{u u}, I_{u d}$ et $I_{d u}$ les intensités correspondant respectivement aux deux flippers à l'arrêt, aux deux flippers en fonctionnement, et à un seul des deux flippers en fonctionnement " $u$ " correspond à "up", associé à une mesure réalisée avec flipper $\pi$; de la même manière, $I_{d}$ est l'intensité mesurée en configuration "down", sans flipper $\pi$ ). Soit $p, f_{1}$ et $f_{2}$ les efficacités respectives du système polariseuranalyseur et des flippers $\pi$, définies par les relations suivantes:

$$
\begin{aligned}
& I_{d d}=(1-p) I_{0} \\
& I_{u t l}=\left(1+f_{1} f_{2} p\right) I_{0} \\
& I_{u d l}=\left(1+f_{1} p\right) I_{0} \\
& I_{d l t}=\left(1+f_{2} p\right) I_{0}
\end{aligned}
$$

Les quatre mesures donnent accès aux quatre grandeurs $I_{0}, p, f_{1}$ et $f_{2}$.

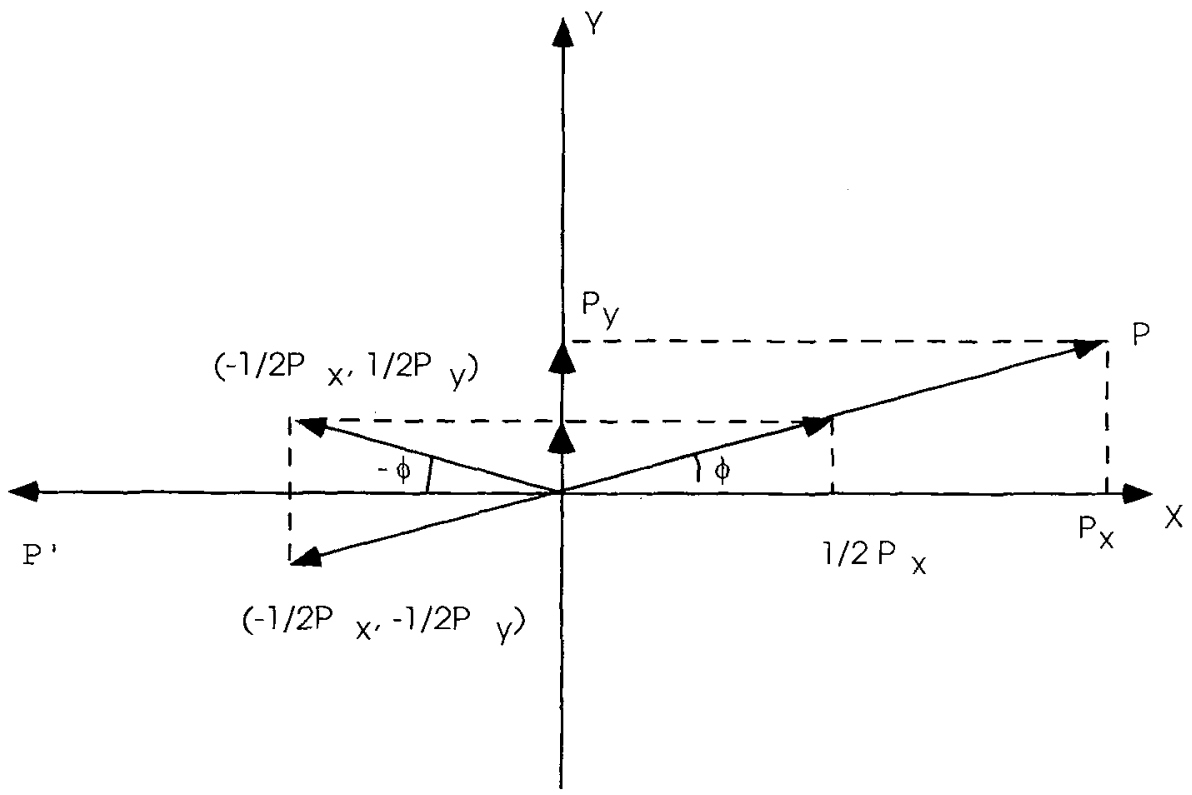

Figure 4: Effet de la diffusion paramagnétique sur les composantes du spin du neutron dans le plan de précession $x-y$. Les neutrons incidents ont une polarisation $\mathrm{P}$. Après diffusion, ta polarisation $\mathrm{P}^{\prime}$ peut être décomposée en deux parties: $\left(-1 / 2 \mathrm{P}_{\mathrm{X}}\right.$ : $\left.1 / 2 \mathrm{P}_{\mathrm{y}}\right)$ est à l'origine de l'écho paramagnétique obtenu sans flipper $\pi ;\left(-1 / 2 \mathrm{P}_{\mathrm{x}},-1 / 2 \mathrm{P}_{\mathrm{y}}\right)$ est équivalent à la polarisation résultant d'une interaction incohérente de spin et ne contribue pas à l'écho en l'absence d'un flipper $\pi$.

Introduisons à présent un échantillon diffuseur sur le trajet des neutrons, et réalisons le même type de mesures mais avec un seul flipper $\pi$ (il en résulte deux valeurs, $I_{u}$ et $I_{d}$ ). L'effet de la diffusion par l'échantillon sur l'état de spin du neutron dépend de la nature de l'interaction. Ainsi la diffusion 
incohérente de spin modifie la polarisation du faisceau incident en renversant deux tiers des spins, alors que la diffusion cohérente non magnétique la laisse inchangée. Dans le cas d'une interaction paramagnétique, un paramètre pertinent est la direction relative du moment magnétique dans l'échantillon par rapport à celles du spin du neutron et du moment transféré. Deux règles essentielles caractérisent cette diffusion:

- Les neutrons ne voient que le magnétisme perpendiculaire à $Q$.

- Le spin du neutron est renversé par une interaction avec un moment magnétique qui lui est perpendiculaire. Il demeure inchangé si le moment magnétique lui est parallèle (voir figure 4).

Il est nécessaire, dans ce cas, de réaliser les deux mesures $I_{u}$ et $I_{d}$ pour trois directions de polarisation différentes du faisceau de neutrons: parallèle à $Q$, perpendiculaire à $Q$ dans le plan de diffusion et verticale.

Le tableau 1 explicite le résultat des différentes mesures $I_{u}, I_{d}$ pour les différents types d'interaction. On peut ainsi, en combinant les différentes relations, déterminer les valeurs de $S_{c o l h}(Q, t=0), S_{i m}(Q, t=0)$ et $S_{\text {magn }}(Q, t=0)$.

\section{Tableau 1.}

\begin{tabular}{|c|c|c|}
\hline $\mathrm{P} / / Q$ & $I_{u}$ & $\frac{(1+p f)}{2} S_{c o h}(Q, t=0)+\frac{(1-p f)}{2} S_{m a g n}(Q, t=0)+\frac{(1-p f / 3)}{2} S_{i n \cdot}(Q . t=0)$ \\
\hline & $I_{d}$ & $\frac{(1-p)}{2} S_{c o h}(Q, t=0)+\frac{(1+p)}{2} S_{m a g n}(Q, t=0)+\frac{(1+p / 3)}{2} S_{i m c}(Q . t=0)$ \\
\hline \multirow[t]{2}{*}{ P perp. $Q$} & $I_{u}$ & $\frac{(1+p f)}{2} S_{c o h}(Q, t=0)+\frac{1}{2} S_{m a g i t}(Q, t=0)+\frac{(1-p f / 3)}{2} S_{i m c}(Q . t=0)$ \\
\hline & $I_{d}$ & $\frac{(1-p)}{2} S_{c o h}(Q, t=0)+\frac{1}{2} S_{\operatorname{magn}}(Q, t=0)+\frac{(1+p / 3)}{2} S_{i n c}(Q, t=0)$ \\
\hline \multirow[t]{2}{*}{$P$ vertical } & $I_{u}$ & $\frac{(1+p f)}{2} S_{c o h}(Q, t=0)+\frac{1}{2} S_{m a g h 1}(Q, t=0)+\frac{\left(1-p f^{\prime} / 3\right)}{2} S_{i n c}(Q, t=0)$ \\
\hline & $I_{d}$ & $\frac{(1-p)}{2} S_{c o h}(Q, t=0)+\frac{1}{2} S_{m a g n}(Q, t=0)+\frac{(1+p / 3)}{2} S_{m}(Q, t=0)$ \\
\hline
\end{tabular}

\subsection{La suite....}

Une expérience typique comprend la détermination de $S(Q, t=0)$, suivie des mesures de $S(Q, t)$ en configuration d'écho, pour différentes valeurs du temps $t$. Les données correspondantes sont normalisées en divisant chaque point $S(Q, t)$ par $S(Q, t=0)$; elles sont ensuite divisées par la fonction de résolution normalisée, mesurée avec un échantillon diffuseur élastique (graphite, quartz).

Les valeurs ainsi obtenues sont généralement comprises entre 0 et 1 . La normalisation par rapport à $S(Q, t=0)$ permet alors, lors des ajustements d'un modèle aux courbes expérimentales, d'imposer la valeur 1 pour $t=0$. Toutefois, si l'échantillon présente un mélange de diffuseurs cohérents et incohérents (par exemple H-D), et si les interactions cohérentes et incohérentes sont associées à des processus de relaxation différents, la normalisation par $S(Q, t=0)$ comme elle a été présentée ci-dessus n'est plus possible. Ceci sera illustré dans l'exemple 2 du chapitre suivant. 


\section{EXEMPLES D'EXPERIENCES SUR UN SES}

\subsection{Mise en évidence de la reptation dans le polyéthylène}

Différentes théories se proposent de décrire la relaxation d'un polymère à chaînes longues, parmi elles le modèle de reptation développé par de Gennes [8].

Avant cette expérience, plusieurs études par diffusion des neutrons avaient été réalisées dans le but de comparer la fonction intermédiaire de diffusion $S(Q, t)$ calculée dans le cadre de ces différentes théories avec les résultats expérimentaux. Malheureusement, la gamme en temps sondée (jusqu'à quelques dizaines de nanosecondes) ne permettait pas de trancher clairement en faveur d'un modèle. La mise au point du spectromètre à échos de spins IN15 à l'ILL [9] étend le domaine en temps accessible jusqu'à 300 ns. Il devenait ainsi possible de mesurer des processus lents de relaxation devant permettre de valider ou invalider les différent modèles.

Il a donc été proposé de mesurer, sur IN15, la dynamique du polyéthylène (PEB-2) fondu $(T=509 \mathrm{~K})$, pour de petites valeurs du moment transféré $\left(0,05 \AA^{-1} \leq \mathrm{q} \leq 0,15 \AA^{-1}\right)$. L'échantillon consistait en un entrelacement de chaînes dont $12 \%$ étaient protonées. L'intensité diffusée comprend trois termes:

- Le premier, cohérent, provient du contraste en longueur de diffusion dû à la dilution de chaînes protonées; il contient l'information relative à la dynamique mono-chaîne.

- Le second, également cohérent, correspond à la diffusion par les chaînes deutérées.

- Le troisième, enfin, est un terme de diffusion incohérente.

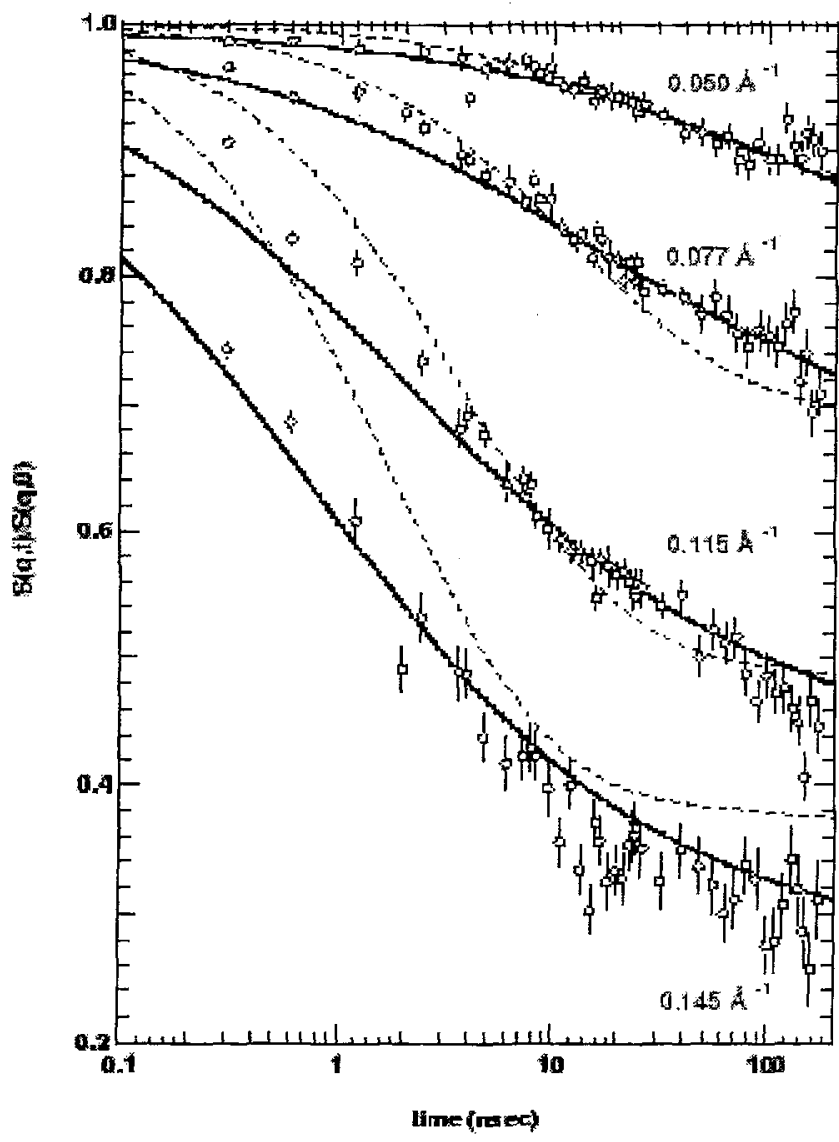

Figure 5: Fonction intermédiaire de diffusion pour différentes valeurs du moment transtéré [10]. 
Comme nous l'avons mentionné plus haut, I'intensité de la diffusion incohérente peut être estimée en réalisant deux mesures up/down $I_{u}$ et $I_{d}$. Elle s'est avérée négligeable.

L'intensité du second terme a été évaluée en mesurant la diffusion par un échantillon similaire, mais purement deutéré. Dès le temps minimum sondé, l'amplitude de l'écho était nulle. Ceci indique que la dynamique cohérente des chaînes est trop rapide pour le domaine de temps de Fourier couvert par l'instrument. Par contre, les chaînes deutérées contribuent à la diffusion intégrée en énergie. Il faut donc corriger la valeur de $S(Q, t=0)$ obtenue pour l'échantillon partiellement hydrogéné, de manière à réaliser une normalisation correcte $S(Q, t) / S(Q, t=0)$ qui permet de fixer à 1 la valeur à $t=0$.

La figure 5 présente quelques exemples de résultats ainsi obtenus. L'ajustement des différents modèles aux données expérimentales a permis de valider clairement la théorie de la reptation [10].

\subsection{Dynamique d'une protéine membranaire}

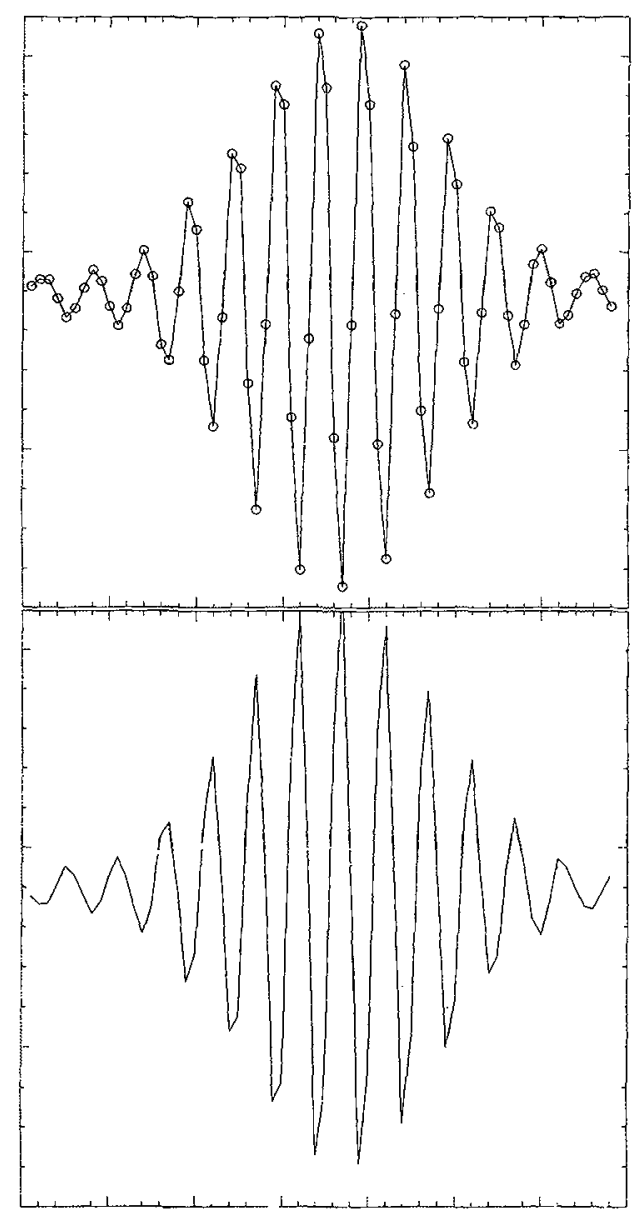

Figure 6: Deux échos déphasés de $\pi$. 
Pour illustrer un peu plus précisément les effets de la présence de différentes contributions cohérentes et incohérentes, nous présentons ici les résultats obtenus avec un échantillon de solvant de protéine. Le spectromètre a été réglé à l'aide d'un échantillon standard diffuseur cohérent élastique. Les mesures réalisées en configuration up/down ont permis de déterminer les valeurs de $S_{\text {coll }}(Q, t=0)$ et $S_{i m}(Q, t=0)$, mettant en évidence un excès de diffusion cohérente. Des mesures d'échos ont ensuite été faites, pour des temps de Fourier allant de 0,012 à $9 \mathrm{~ns}$. Sur la première décade, les échos enregistrés indiquent un déphasage de $\pi$ de l'angle de précession $\varphi$, comme on peut le voir sur la figure 6 .

Ceci signifie que les spins participant à l'écho ont été renversés par l'interaction avec l'échantillon, et donc que entre $0,012 \mathrm{~ns}$ et $0,10 \mathrm{~ns}$, les corrélations dominantes sont incohérentes. Vers $0,10 \mathrm{~ns}$. l'amplitude disparaît puis un écho réapparât aux temps plus longs, sans décalage de phase. Il est associé à un second processus de diffusion cohérent et plus lent. L'évolution schématique de l'écho en fonction du temps est illustrée sur la figure 7.

Dans un tel cas, il est impossible de normaliser par rapport à $S(Q, t=0)$. Il faut en effet se souvenir ici que le principe des échos de spins repose, à la base, sur l'utilisation d'un faisceau de neutrons polarisé. Si l'interaction avec l'échantillon dépolarise le faisceau, la modulation d'intensité présentée sur la figure 2 disparaît. Dans le cas de la présence de différents processus de diffusion cohérents et incohérents, pou chaque valeur du temps $t$ sondé l'amplitude maximale de l'écho est donnée par $\left(S_{(t) h}(Q, t)-S_{i m}(Q, t) / 3\right)$. Ceci n'est autre que $I_{u}(t)-I_{d l}(t)$ où $I_{t r}(t)$ et $I_{t}(t)$ sont les grandeurs définies dans le tableau 1 généralisées à des valeurs quelconques du temps $t$ - pour simplifier, on considère ici un SES idéal pour lequel $p, f$ ainsi que la fonction de résolution normalisée sont égaux à 1-. Dans l'exemple présent, $\left(S_{r \prime m}(Q, t)-S_{i m c}(Q, t) / 3\right)$ $s^{\prime}$ annule vers $t=0,10 \mathrm{~ns}$. Aux temps plus courts, la diffusion incohérente est en excès et est ì l'origine des échos déphasés de $\pi$; au-delà de $0,10 \mathrm{~ns}$, la diffusion cohérente devient prépondérante et l'écho réapparaît, sans déphasage.

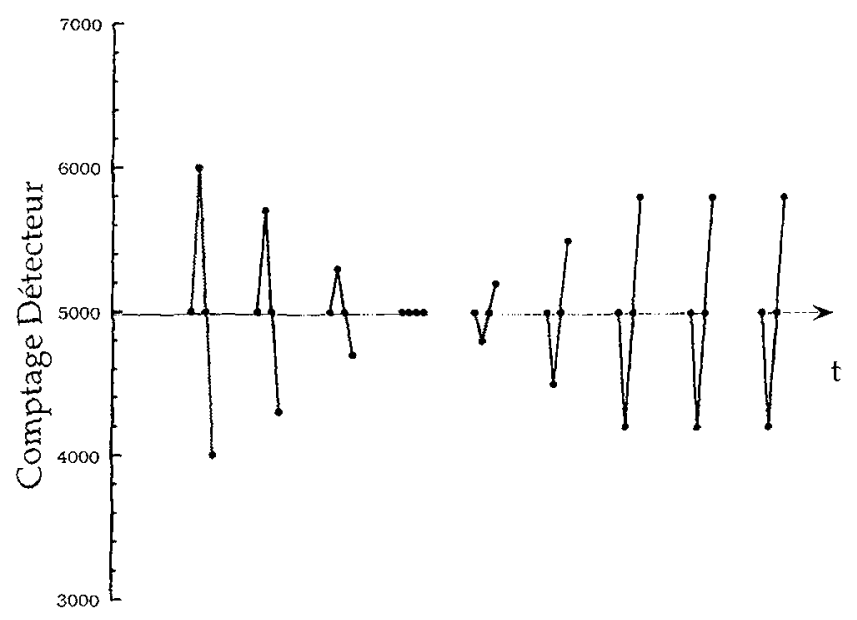

Figure 7: Evolution de l'amplitude de l'écho en fonction du temps $t$, dans le cas de l'exemple décrit au paragraphe IV-2. Pour chaque valeur sondée du temps $t$, l'amplitude est déterminée en mesurant quatre points déphasés de $\pi / 2$, obtenus en variant le courant dans la bobine de phase aurour de la valeur d'équilibre des deux intégrales de champ. Le réglage réalisé avec un échantillon diffuseur cohérent élastique était lel que les quatre points décrivaient la fonction $A \sin (j-\pi / 2)+B$, avec respectivement les valeurs $j=-\pi / 2,0 . \pi / 2$ el $\pi$. C est le ciss des échos mesurés aux temps les plus longs, et donc associés à un processus de diffusion colnérente. Par contic. aux temps courts, on observe un déphasage de $\pi$. Ces échos proviennent de la diffusion incohérente des neutrons par l'échantillon, qui est dominante dans ce domaine de temps. 


\subsection{Dynamique d'un composé magnétique désordonné}

Un verre de spin réentrant est un composé magnétique qui, lorsque la température décrôt, passe d'une phase paramagnétique désordonnée à une phase magnétiquement ordonnée, puis, à plus basse température encore, subit une seconde transition de phase vers un état caractérisé par un désordre de type verre de spin. Le composé $\mathrm{CdCr}_{2} \mathrm{~S}_{4}$ est un ferromagnétique simple pour des températures inférieures à $84 \mathrm{~K}$. Du fait de la frustration présente dans le réseau magnétique, la substitution d'ions non magnétiques $\mathrm{In}^{3+}$ aux ions magnétiques $\mathrm{Cr}^{3+}$, entraîne l'apparition d'une phase verre de spin réentrante pour des valeurs de la dilution inférieures à $10 \%$. Au delà, les composés sont des "vrais" verres de spin .

Le but de cette expérience était d'étudier la dynamique des spins dans le composé $\mathrm{CdCr}_{2(1-x)} \mathrm{In}_{2 \mathrm{x}} \mathrm{S}_{4}$ $(x=0,10)$, qui ne présente pas d'ordre ferromagnétique à longue distance. On a donc adopté la configuration d'échos de spins paramagnétique; c'est une configuration standard sans flipper $\pi$. En effet, comme on peut le voir sur la figure 4 , la polarisation du faisceau après interaction avec un échantillon paramagnétique peut être décomposée en deux parties. $\left(-1 / 2 \mathrm{P}_{\mathrm{x}}, 1 / 2 \mathrm{P}_{\mathrm{y}}\right)$ correspond à une transformation de $\varphi$ en $-\varphi$, équivalente à celle réalisée par un flipper $\pi \cdot\left(-1 / 2 \mathrm{P}_{\mathrm{x}},-1 / 2 \mathrm{P}_{\mathrm{y}}\right)$ est associé à un déphasage de $\pi$ qui, en présence d'un flipper $\pi$, donnerait un écho similaire à celui présenté dans l'exemple précédent. Ainsi, on obtient, en l'absence de flipper $\pi$, un écho d'amplitude maximale $1 / 2 S_{\text {magn }}(Q, t=0)$.

Un avantage important de cette technique est d'éliminer, dans les mesures d'échos, toute contribution autre que magnétique. Mais la normalisation doit donc être réalisée par rapport à la partie magnétique seule $S_{\text {magn }}(Q, t=0)$. Comme nous l'avons vu, la détermination de cette grandeur nécessite une mesure des intensités $I_{u}$ et $I_{d}$ pour trois directions de la polarisation du faisceau de neutrons : verticalement, parallèlement à $Q$ et perpendiculairement à $Q$.

La figure 8 montre un exemple de courbes obtenues. Nous avons pu mettre en évidence deux processus de relaxation liés respectivement aux caractères ferromagnétique et verre de spin du composé étudié [11].

La figure 9 présente un exemple de courbes obtenues dans le cadre de l'étude des fluctuations superparamagnétiques dans un échantillon constitué de particules de Fer dispersées dans une matrice d'alumine. Les mesures ont été réalisées sur les spectromètres IN11 et IN15 utilisés en configuration paramagnétique [12].

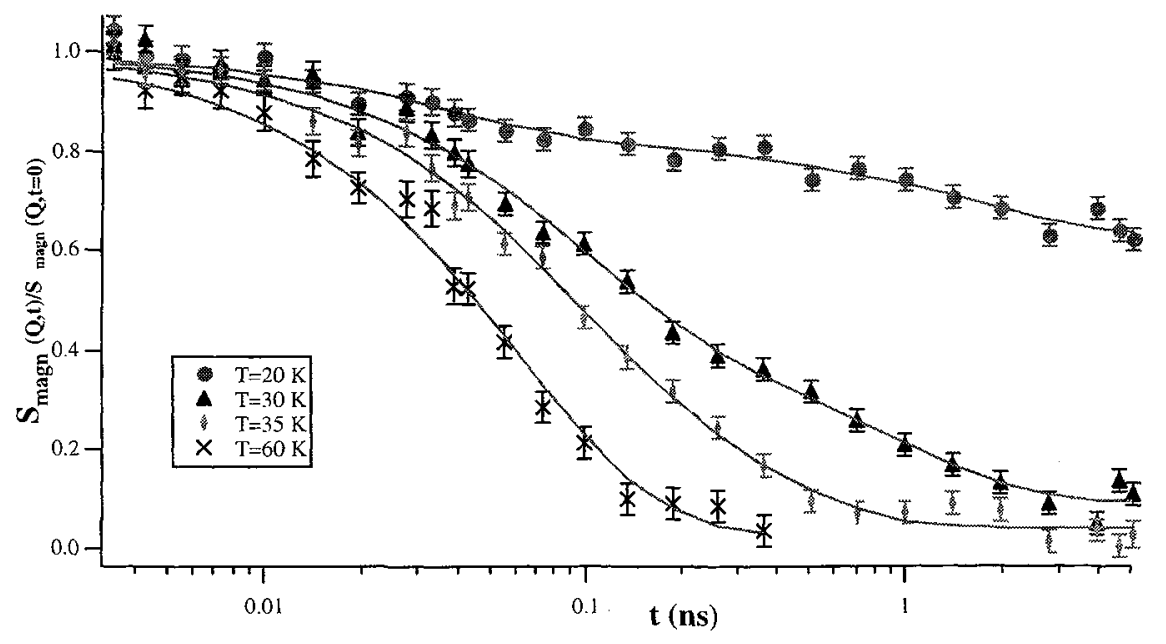

Figure 8: $\mathrm{CdCr}_{1,8} \operatorname{In}_{0.2} \mathrm{~S}_{4}$ - Fonction intermédiaire de diffusion mesurée à différentes températures pour $\mathrm{Q}=0,066 \AA^{-1}$. Les lignes sont le résultat de l'ajustement d'un modèle exponentiel pour $T=60 \mathrm{~K}$ et $35 \mathrm{~K}$, et de lá somme de deux exponentielles: pour les deux autres températures [11]. 


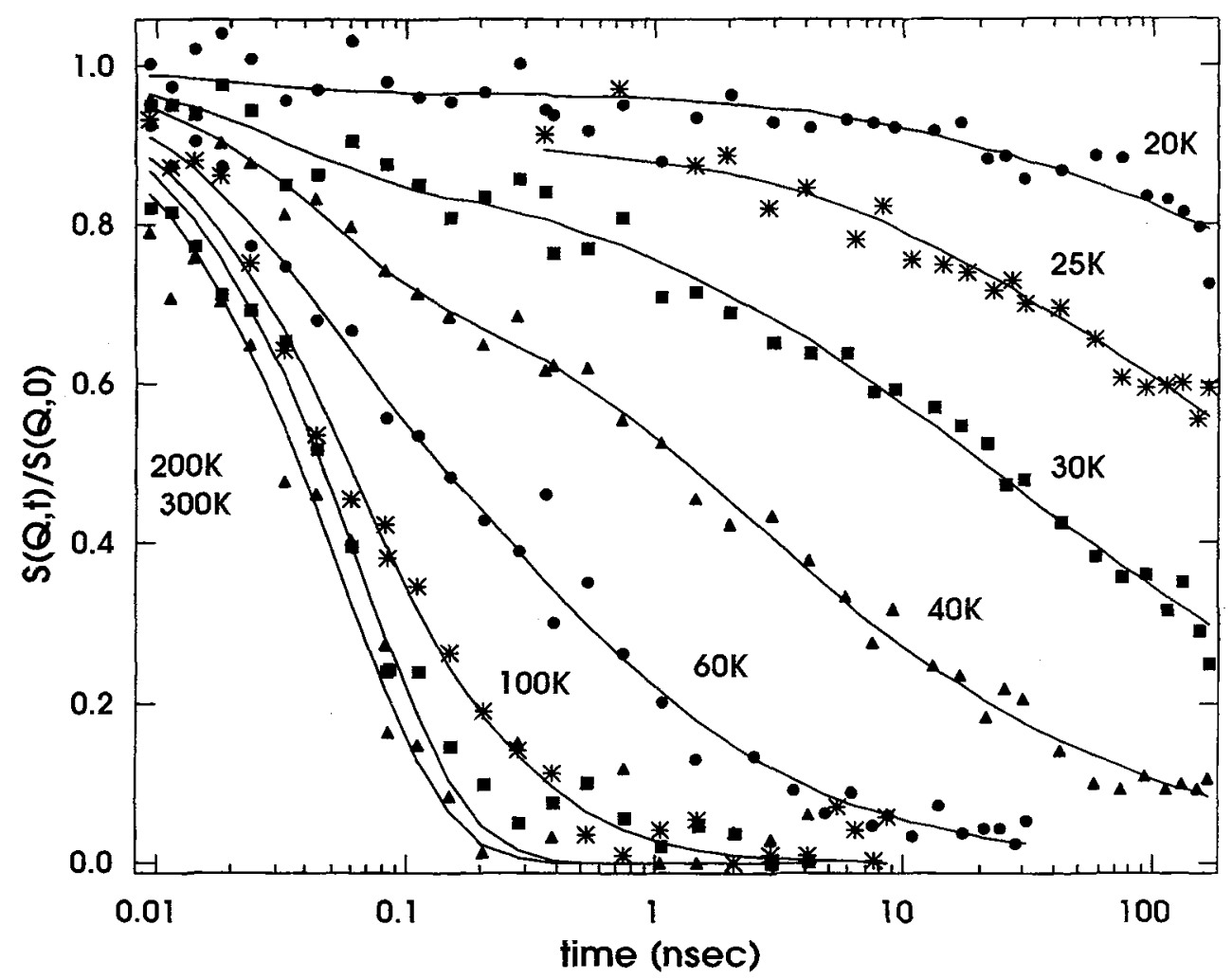

Figure 9: $\mathrm{Fe}$ dans $\mathrm{Al}_{2} \mathrm{O}_{3}$. Evolution, en fonction de la température, de la fonction intermédiaire de diffusion nommaliscé mesurée à $Q=0,07 \AA^{-1}$ - données combinées des SES IN 1$]$ el IN15 - [12].

\section{REVUE DES SES ACTUELLEMENT DISPONIBLES}

IN11 fut le premier SES en service. Depuis sa mise au point, il a beaucoup évolué, et fait des petits...Nous allons ici passer en revue les différents spectromètres actuellement disponibles, en donnant brièvement leurs caractéristiques.

\subsection{IN11 (ILL)}

Angle de diffusion $(2 \theta)$ :

Longueur d'onde:

Intégrale de champ maximale:
$3-120$ degrés

$4,5-10$ Angströms

276460 Oe.cm

L'utilisation d'une configuration dite de petit écho, pour laquelle l'aire de précession est réduite. permet de couvrir trois ordres de grandeur en temps de Fourier. Ainsi, avec une longueur d'onde de 6 Angströms, IN11 donne accès à des temps compris entre la dizaine de picosecondes el 11 nanosecondes, pour des moments transférés accessibles allant de 0,055 à $1,8 \AA^{-1}$. 
Une option "multi-détecteurs" est également disponible (IN11C) [13]. On dispose alors de 41 détecteurs, qui couvrent un secteur angulaire de 30 degrés. L'angle de diffusion minimum est alors 20 degrés, et l'intégrale de champ est actuellement limitée à 45240 Oe.cm. Cette configuration. opérationnelle depuis 18 mois à peu près, a récemment été utilisée, avec beaucoup de succès, pour l'étude de la transition vitreuse dans la toluidine, de la diffusion de l'eau lourde surfondue dans le vycor, ainsi que pour celle de polyélectrolytes hydrogénés. Dans ce dernier cas, le facteur trois de réduction de l'intensité lié à la nature incohérente de la diffusion a été largement compensé par l'utilisation des 41 détecteurs.

\subsection{IN15 (ILL)}

IN 15, à l'ILL, est un SES à très haute résolution qui, dans sa configuration standard, utilise des longueurs: d'onde comprises entre 8 et $20 \AA$. Il permet d'augmenter d'environs un ordie de grandeur le temps de Fourier maximum accessible avec IN 1 1, approchant ainsi la microseconde. Il peut accéder à des moments transférés allant de 0,01 et $0,8 \AA$ A- 1 . Deux options supplémentaires sont également prévues:

- Un miroir focalisant pour une utilisation aux grandes longueurs d'onde $(17-15 \AA)$ avec une haute résolution en $Q$. Il permet un gain important en intensité par rapport à l'utilisation de collimateurs.

- Un fonctionnement en mode Temps de Vol est en cours de développement. Il permettra de réalisel des échos à partir d'impulsions de neutrons délivrées par un système de choppers. La mise au point est complexe, nécessitant par exemple de coupler la variation du courant dans les flippers au temps de vol des neutrons de l'impulsion. Cette option devrait permettre de couvrir, en un spectre, un domaine plus étendu de temps de Fourier. Elle sert également de test en vue du développement de la technique d'échos de spins sur les sources à neutrons pulsées.

\subsection{MESS (LLB)}

Moment transféré:

Longueur d'onde:

Temps de Fourier:

$$
\begin{aligned}
& 0,027-0,25 \AA-1 \\
& 5-8 \text { Angströms } \\
& 1-40 \text { nanosecondes }
\end{aligned}
$$

Du fait de la gamme réduite en moments transférés accessibles, ce spectromètre a une thématiçue typiquement petits angles [14]. Les sujets les plus couramment étudiés sont

- en physicochimie: phases éponges, micro-émulsions, colloïdes.

- en biologie: régime de diffusion de protéines en solution.

\subsection{SPAN (HMI)}
Angle de diffusion:
$2-150$ degrés
Longueur d'onde:
$2,5-10$ Angströms
Intégrale de champ maximale: 90000 Oe.cm

Un nouveau spectromètre à échos de spins vient d'être mis en service au HMI (Berlin). Son originalité repose dans la symétrie axiale du champ de précession, qui permet de mesurer simultanément sur un grand domaine angulaire. Ce champ est créé par trois paires de bobines. Dans chaque paire, les deux bobines sont placées de part et d'autre du plan de diffusion, leurs centres et celui de l'échantillon étant portés par le même axe vertical. 
Un mode de fonctionnement en spectromètre à temps de vol est également disponible, avec une résolution en énergie de l'ordre de $0,1 \mathrm{meV}$ à $7 \AA$. Combinée aux mesures d'échos de spin, il donne accès à un domaine très large de transferts d'énergie.

\section{Références}

[1] D. Richter et al, Phys. Rev. Lett. 41, 1484, 1978. Rathgeber et al, Physica B 234-236, 258, 1997.

[2] Wuttke et al, J. Chem. Phys. 105, 5177, 1996. Prevel et al, J. Phys. Cond. Matt. 6, 1279, 1994.

[3] F. Mezei et al., J. Magn. Magn. Mat. 14, $211,1979$. C. Pappas et al., J. Appl. Phys. 79, 6158, 1996. Voir également [10] et [11].

[4] B. Farago, Physica B 226, 51, 1996.

[5] F. Mezei, Phys. Rev. Lett. 44, 1601, 1980.

[6] F. Mezei, in: Neutron Spin Echo, Lecture Notes in Physics, vol. 122, Springer, Berlin 1980.

[7] C. Cohen-Tannoudji, Bernard Diu et Franck Laloë, Mécanique Quantique Tome 1.

[8] P.G. De Gennes, J. Chem. Phys. 55, 572, 1971.

[9] P. Schleger et al, Physica B 241-243, 164, 1998.

[10] P. Schleger et al, Phys. Rev. Lett. 81,124,1998.

[11] S. Pouget et al., Physica B 267-268, 304-307, 1999.

[12] H. Casalta et al, Phys. Rev. Lett., sous presse.

[13] B. Farago, Physica B 241-243, 113, 1998.

[14] R. Papoular, Thèse de Doctorat, 1992. 